\title{
Generality of US preexposure effects: Effect of shock or food preexposure on water escape
}

\author{
TAMIR CASPY and REUVEN FROMMER \\ Bar-Ilan University, Ramat-Gan, Israel \\ and \\ INA WEINER and R.E. LUBOW \\ Tel-Aviv University, Ramat-Aviv 420111, Israel
}

\begin{abstract}
Mice preexposed to nonresponse-contingent shock offset or nonresponse-contingent food delivery performed more poorly in a water-escape task than groups receiving no preexposures or preexposure to controllable shock or controllable food. It was concluded that US preexposure procedures produce a very broad generalization of effects. These results are discussed in relation to similar findings in the literature.
\end{abstract}

Animals preexposed to a series of unavoidable and inescapable (response-independent) shocks exhibit marked interference in subsequent avoidance or escape behavior. Conversely, animals receiving the same amount of escapable (response-contingent) shock show no such interference. This phenomenon is known best under the name of "learned helplessness" (for a recent review, see Maier \& Seligman, 1976).

One of the questions of interest in the investigation of learned helplessness is that of its generality. Typically, in the learned helplessness studies, preexposure to shock and the subsequent test are conducted in different chambers. Therefore, it is clear that the interference effect generalizes, at least, to an environment that is different from the preexposed one.

Although the typical learned helplessness procedure has different environments for the preexposure and test phases, it employs the same noxious stimulus, namely shock, in both phases. Braud, Wepman, and Russo (1969) were the first to show that the interference effect generalizes not only across environments but also across different aversive tasks. Mice preexposed to inescapable shock later failed to acquire a water-escape response, as compared to mice that could escape from shock. Thus, generalization of the debilitative effect of inescapable shock preexposure was obtained with different aversive preexposure and test stimuli, shock vs. water, and with different response requirements during preexposure and test, jumping vs. swimming.

In a study by Altenor, Kay, and Richter (1977), half of the rats were preexposed to shock and half to water. On the test, half of each group was run on a water-escape task and half on a shock-escape task. As

Reprints may be obtained from R. E. Lubow, Department of Psychology, Tel-Aviv University, Ramat-Aviv, Israel. long as the preexposure condition contained the element of inescapability, the learned helplessness effect was found. Furthermore, there was as much of a decrement in performance in transferring from water to shock or shock to water as there was when the aversive stimulus was the same in the preexposure and test phases.

A number of investigators have reported data that represent an appetitive parallel to the learned helplessness phenomenon (Engberg, Hansen, Welker, \& Thomas, 1972; Welker, 1976). Animals preexposed to responseindependent food presentation were later retarded in acquiring a response to obtain food. In an extensive study by Goodkin (1976), response and reinforcement generalizations were tested using both appetitive and aversive tasks in preexposure. Six groups of rats were preexposed as follows: One group was trained to nosepress for food, a second to chain-pull for food, a third to chain-pull to escape shock, and a fourth and fifth group received no treatment. All groups were tested in a nose-press escape-avoidance task. The three groups with prior contingent response training rapidly acquired the response, while the two groups that received response-independent preexposure failed to do so. Goodkin concluded that the critical factor in producing the learned helplessness effect is the relationship between response and reinforcement (lack of contingency) and not the aversive quality of the preexposed stimulus interacting with lack of contingency.

The purpose of the present experiment was to extend the findings of cross-motivational interference effects, using within one experiment both appetitive and aversive preexposure and testing with a common, unrelated task.

\section{METHOD}

Subjects

Seventy-two naive male BALB-C mice, obtained from the 
Bar-Ilan University vivarium, were randomly assigned to one of six groups. Each animal was housed individually for 1 week prior to, and for the duration of, the experiment. At the beginning of the experimental procedure, the animals were between 56 and 63 days of age.

\section{Apparatus}

The preexposure phase of the experiment was conducted in six identical $16 \times 16 \mathrm{~cm}$ Plexiglas chambers. A microswitch lever, $17 \times 3 \mathrm{~mm}$, requiring $10 \mathrm{~g}$ force to activate, protruded from one wall of the chamber. A food cup attached to a pellet dispenser (David Scientific Instrument, Model PD 104) was located alongside the lever. The chamber floor was composed of stainless steel bars, $.6 \mathrm{~cm}$ in diameter, with a center-to-center distance of $.9 \mathrm{~cm}$. The grid was connected to a scrambled constant-current shock source.

Each chamber was placed individually in a sound-attenuated ventilated cubicle. All illumination within the chamber came from an $18 \times 17.5 \mathrm{~cm}$ cubicle window, covered with red cellophane, facing onto a normally lit room.

The test was conducted in a $77 \times 50 \times 40 \mathrm{~cm}$ water tank. Approximately $2 \mathrm{~cm}$ from one of the end walls of the tank, and parallel to it, a metal ramp $18 \times 25 \mathrm{~cm}$ extended into the water such that $6 \mathrm{~cm}$ of it was submerged and $12 \mathrm{~cm}$ was out of the water. The ramp made a 30-deg angle with the tank wall. The dry end of the ramp ended on a small $(10 \times 10 \mathrm{~cm})$ horizontal platform that extended outside of the tank. A photoelectric cell was located on the platform, $2.0 \mathrm{~cm}$ from the tank edge and $1.0 \mathrm{~cm}$ above the platform. Water temperature was relatively constant at $25^{\circ} \mathrm{C}$.

\section{Procedure}

Six animals, one from each group, were run simultaneously in the preexposure phase. This phase was conducted over 5 days, in 1-h daily sessions. During this time each animal was placed in the chamber and treated according to its group designation.

Group CO-s subjects were presented with shocks that they could terminate. A .2-mA shock with a maximum duration of $10 \mathrm{sec}$ was delivered to the grid floor. The intertrial interval, measured from the termination of one shock to the onset of the next shock, was 20 sec. A leverpress during the shock terminated it.

Group UNCO-s subjects received uncontrollable shock. UNCO-s subjects were yoked to CO-s subjects such that when a subject from the latter group pressed the lever and terminated the shock, the shock was also ended for its yoked partner.

Group N-s subjects were treated in a manner identical to the first two groups, except that they did not receive shock.

Subjects of the next three groups were placed on a $23-\mathrm{h}$ deprivation schedule for 7 days prior to the preexposure session. They were maintained on this schedule throughout the duration of the experiment.

Group CO-f subjects controlled food delivery. They were given five daily $1-\mathrm{h}$ sessions in which each barpress delivered a 45-mg Noyes pellet.

Group UNCO-f subjects had no control over food delivery. UNCO-f subjects were yoked to CO-f subjects such that when a subject from the latter group pressed the bar and received food, food also was delivered to its yoked partner.

Group N-f subjects were treated in a manner identical to Groups CO-f and UNCO-f, except that they did not receive food pellets in the experimental chamber.

Immediately after each daily session, all food-deprived subjects received $1 \mathrm{~h}$ of free access to food in their home cages. Number of barpresses per session was recorded for Groups CO-s, UNCO-s, CO-f, and UNCO-f.

On Day 6, subjects from all groups were given five trials in the water-escape test. The mouse was held by the tail and gently lowered into the water in the corner of the tank, opposite the ramp. When it touched the water, a timer was started. The timer stopped when the mouse climbed up the ramp and broke the photoelectric beam. If an animal did not escape within 2 min, the trial was manually terminated and a 2 -min score was recorded. The intertrial interval, measured from the end of one trial to the beginning of the next, was $1 \mathrm{~min}$. The experiment was run over a period of 3 weeks in six replications, two subjects per group in each replication. The running order was randomized.

\section{RESULTS}

The mean number of barpresses as a function of session for the four groups for which this measure was recorded is shown in Figure 1. It is quite clear that both groups that had control over the reinforcement learned to exercise that control. A three-way ANOVA, control vs. no control, shock vs. food, and the repeated measure of sessions, indicated that all three factors produced highly reliable differences $[F(1,44)=304.76, p<.001$; $F(1,44)=45.18, p<.001 ; F(4,176)=125.12, p<.001]$. The three two-way interactions and the three-way interaction also were highly significant (ps $<.001)$.

The mean escape latency for all six groups as a function of trials is shown in Figure 2. The latency data were analyzed with a 3 by 2 by 5 ANOVA. The first factor represented the $\mathrm{CO}$, UNCO, and NO groups, the second factor represented the food and shock groups, and the third was a repeated measure for the five trials. While the control variable gave reliable differences $[F(2,66)=20.56$, $\mathrm{p}<.001]$, there were no such differences for the foodshock variable $[F(1,66)=1.78, p>.10]$. The trials effect was significant $[F(4,264)=55.65, p<.001]$, as were the interactions between trials and food vs. shock $[\mathrm{F}(4,264)=2.56, \mathrm{p}<.05]$ and between trials and control $[F(8,264)=2.85, p<.01]$. It is apparent from Figure 2 that both interactions are a result of the CO-f group exhibiting a relatively high latency on the first trial.

Differences between the CO, NO, and UNCO groups were analyzed by Tukey's method of contrasts. There was a significant difference between $\mathrm{CO}$ and UNCO

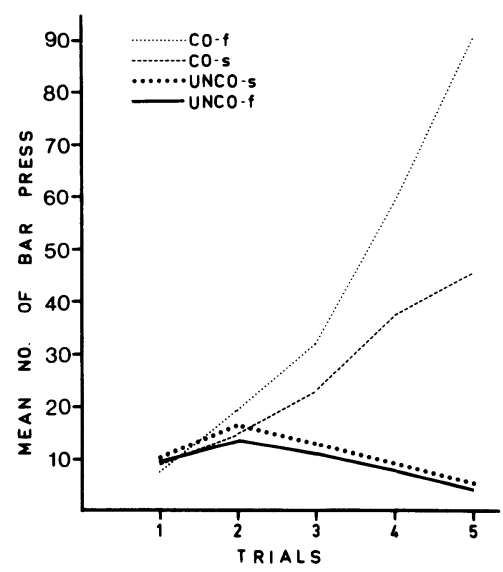

Figure 1. Mean number of barpresses for each of four groups: control of food (CO-f), noncontrol of food (UNCO-f), control of shock (CO-s), and noncontrol of shock (UNCO-s). 


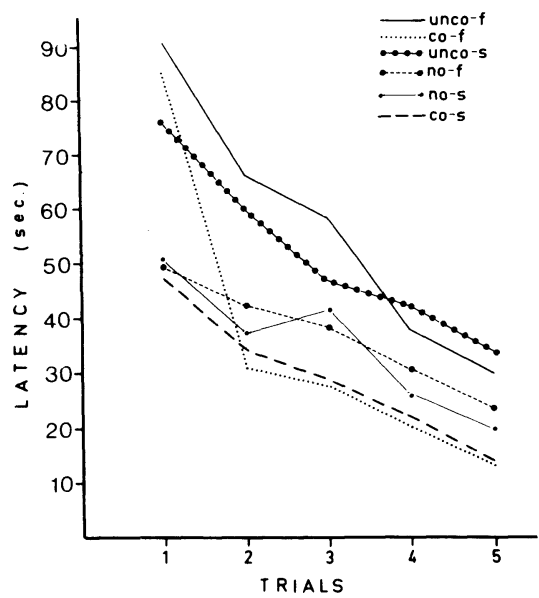

Figure 2. Mean escape latencies for all six groups: control of food (CO-f), noncontrol of food (UNCO-f), no food (NO-f), control of shock (CO-s), noncontrol of shock (UNCO-s), and no shock (NO-s).

$(\mathrm{p}<.01)$ and between $\mathrm{N}$ and UNCO $(\mathrm{p}<.01)$, but not between $\mathrm{CO}$ and $\mathrm{N}(\mathrm{p}>.05)$. These differences, collapsed across the food-shock variable, are shown in Figure 3.

\section{DISCUSSION}

The major finding of this study was that the lack of contingency, as expressed in those groups that did not have control over food delivery or shock offset (UNCO-f, UNCO-s) during exposure, produced a performance decrement on the subsequent swim test as compared to those groups with control (CO-f, CO-s) or with no preexposure. These results are similar in a number of ways to those obtained in other studies.

The detrimental effects of uncontrollable shock, commonly called "learned helplessness," have been repeatedly demonstrated, although mostly in a shock-based escape-avoidance task (see Maier \& Seligman, 1976, for a review). However, there are a few studies that also demonstrate transfer of the negative effects of shock preexposure to nonshock-based tests such as swimming (e.g., Altenor et al., 1977; Braud et al., 1969).

Likewise, detrimental effects of uncontrollable food,

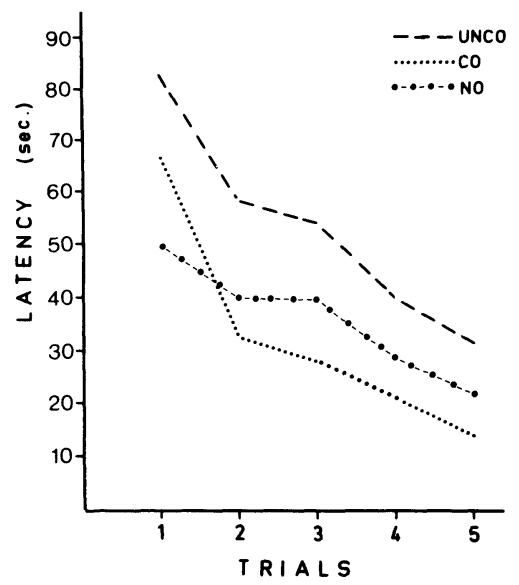

Figure 3. Mean escape latencies for control, noncontrol, and NO groups collapsed across the food-shock variable. sometimes called "learned laziness," have been demonstrated, although mostly on the acquisition of food-reinforced instrumental responses (e.g., Engberg et al., 1972; Welker, 1976). However, here too, it has been shown that there is negative transfer to an unrelated task. Specifically, Goodkin (1976) reported that rats preexposed to noncontingent food showed retarded acquisition on a subsequent shock avoidance-escape task. Goodkin also demonstrated that control over shock offset or food delivery during the preexposure period enhanced test performance for experimental animals as compared to naive controls. This finding is not supported in the present study, where it was found that there was no difference between the test scores of the nonpreexposed groups and those groups preexposed to controllable food or shock.

Although this inconsistency between the two studies might be related to the different species employed, rats vs. mice, there are other procedural dissimilarities that are more likely involved in producing the discrepancy. Each of Goodkin's (1976) major groups was preexposed to the apparatus for different periods of time. Thus the zero-preexposure group had no familiarity with the apparatus before the escape-avoidance test, the no-control group had 15 days of preexposure, and the groups that controlled shock or food delivery had 7 days of apparatus preexposure. From a variety of studies, one might expect that the amount of apparatus preexposure before administering a learning task in the same apparatus would be positively related to the performance on that task. (See the old literature on latent learning, in particular, Types I and II as described by Thistlethwaite, 1951; more recently, Grant \& Young, 1971; Posluns \& Vanderwolf, 1970). Indeed, at least logically, there might well be an interaction between the confounding variable of amount of apparatus preexposure and type of preexposure (none, no control, and control) that may have affected the entire pattern of results.

The present study eliminated this possible source of confounding. All groups had the same amount of apparatus preexposure. The results support Goodkin (1976) on the major conclusions concerning generality, but fail to support him in regard to better performance of stimulus preexposure with control as compared to no preexposure.

The present study, then, complements and extends the findings of Altenor et al. (1977), Braud et al. (1969), and Goodkin (1976). First of all, the effects obtained in the present study parallel, within one study, what has been concluded from separate research under different conditions. Noncontingent preexposure to either a negative US (shock) or a positive US (food) results in depressed performance on a task that is unrelated to either of the preexposure conditions (swim test).

These results support the conclusion that there is extensive generality and generalization of US preexposure effects. It would appear that the decremental effects depend on neither the quality of the stimulus that is preexposed nor the similarities between the preexposed stimulus and stimuli used in the test.

\section{REFERENCES}

Altenor, A., Kay, E., \& Richter, M. The generality of learned helplessness in the rat. Learning and Motivation, 1977, 8, 54-61.

BraUd, W., WePMAN, B., \& Russo, D. Task and species generality of the "helplessness" phenomenon. Psychonomic Science, 1969, 16, 154-155.

Engberg, L. A., Hansen, G., Welker, R. L., \& Thomas, D. R. Acquisition of key-pecking via autoshaping as a function of prior experience: "Learned laziness"? Science, 1972, 178, 1002-1004.

Goodkin, F. Rats learn the relationship between responding and environmental events: An expansion of the learned helplessness hypothesis. Learning and Motivation, 1976, 7, 382-393.

Grant, M., \& Young, D. The effects of preexposure to a learning 
apparatus. Behavioral Research Methods \& Instrumentation, 1971, 3, 251-252.

Maier, S. F., \& Seligman, M. E. P. Learned helplessness: Theory and evidence. Journal of Experimental Psychology: General, 1976, 105, 3-46.

Posluns, D., \& VANDe RWolf, C. H. Improved avoidance performance following exploratory movement. Canadian Journal of Psychology, 1970, 24, 499-504.
Thistlethwaite, D. A critical review of latent learning and related experiments. Psychological Bulletin, 1951, 48, 97-129.

WELKE R, R. L. Acquisition of a free operant appetitive response in pigeons as a function of prior experience with responseindependent food. Learning and Motivation, 1976, 7, 394-405.

(Received for publication March 26, 1979.) 